\title{
Recommendations for the assessment of non-extremity venous thromboembolism outcomes: communication from the SSC of the ISTH
}

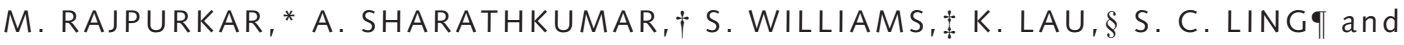 \\ A. K. C. CHAN, ${ }^{*}$ FOR THE SUBCOMMITTEE ON PEDIATRIC/NEONATAL HEMOSTASIS AND \\ THROMBOSIS \\ *Division of Pediatric Hematology Oncology, Carmen and Ann Adams Department of Pediatrics, Children's Hospital of Michigan, Wayne \\ State University, Detroit, MI; †Ann \& Robert H. Lurie Children's Hospital of Chicago, Northwestern University Feinberg School of Medicine, \\ Chicago, IL, USA; †Division of Haematology Oncology, The Hospital for Sick Children, University of Toronto, Toronto; §Division of Pediatric \\ Nephrology, McMaster University, Hamilton; @Division of Gastroenterology, Hepatology \& Nutrition, Department of Paediatrics, The Hospital \\ for Sick Children, University of Toronto, Toronto; and * Division of Haematology Oncology, McMaster Children's Hospital, McMaster \\ University, Hamilton, Ontario, Canada
}

To cite this article: Rajpurkar M, Sharathkumar A, Williams S, Lau K, Ling SC, Chan AKC, for the Subcommittee on Pediatric/Neonatal Hemostasis and Thrombosis. Recommendations for the assessment of non-extremity venous thromboembolism outcomes: communication from the SSC of the ISTH. J Thromb Haemost 2015; 13: 477-80.

\section{Introduction}

Post-thrombotic syndrome (PTS) is an important outcome after venous thromboembolism (VTE), and recommendations for standardized measurements following extremity VTE have been published [1,2]. However, the clinical spectrum of pediatric VTE extends beyond the extremities in approximately $25-30 \%$ of children, and long-term outcomes after non-extremity VTE have not been measured. As such outcomes are site-dependent and the clinical spectrum is heterogeneous, currently available PTS outcome scales are inadequate for the measurement.

To address this issue, the Perinatal and Pediatric Hemostasis Subcommittee of the ISTH established a working group (WG) to make recommendations for uniform definitions of non-extremity VTE outcomes. Although the original plan was to review the available literature and develop guidelines, it was realized that there is a paucity of literature in this area, and recommendations were therefore

Correspondence: Madhvi Rajpurkar, Division of Pediatric Hematology Oncology, Carmen and Ann Adams Department of Pediatrics, Children's Hospital of Michigan, Wayne State University, Detroit, MI 48201, USA.

Tel.: +1 313745 5515; fax: + 13137455237 .

E-mail: mrajpurk@med.wayne.edu

Received 16 May 2014

Manuscript handled by: S. Eichinger

Final decision: F. R. Rosendaal, 28 November 2014 developed on the basis of a review of the available literature and by consensus. The recommendations were presented at the 60th SSC meeting in Liverpool, 2012, and a manuscript draft was developed by the WG which was then circulated to the Subcommittee chairman and subsequently revised to incorporate the input.

\section{Overview of WG discussion}

Definition and scope of the problem:

The members concurred with the recommendation from the WG on extremity PTS that the complications following non-extremity thrombosis do not fall within the scope of the definition of typical PTS, and thus the term 'post-thrombotic sequelae' was more appropriate for describing such outcomes [1,2].

Inclusion of the spectrum of sites for non-extremity thrombosis:

According to the WG, the term 'non-extremity thrombosis' should encompass these sites:

- Intra-abdominal: Renal vein thrombosis (RVT) Portal vein thrombosis (PVT)

- Pulmonary arterial thrombosis (pulmonary embolism [PE])

- Central nervous system (CNS): cortical sinus venous thrombosis (CSVT)

- Rare sites: mesenteric vein thrombosis, pelvic veins (ovarian or uterine) thrombosis, hepatic vein thrombosis (Budd-Chiari syndrome [BCS]), and retinal vein thrombosis 
Inferior and superior vena caval thrombosis is often associated with extremity thrombosis, and the WG therefore recommended that complications after caval thrombosis should be included with extremity thrombosis outcomes. Also, the modified Villalta scale has a provision to incorporate long-term complications of superior vena caval thrombosis, and may be used [3]. The WG also decided to exclude outcomes after CSVT, as a separate WG will focus on defining outcomes of CNS thrombosis.

\section{Recommendations for follow-up:}

The WG recommended that patients should be followed at specific time points, with at least annual evaluation after the thrombotic event. It was also acknowledged that such evaluations, ideally, should be performed by the patient's primary-care physicians. However, as uniform data on the outcomes are not available, the committee felt that, until this becomes the standard of care, such evaluation(s) should preferably be performed by the primary subspecialty following the organ dysfunction, with close communication with the primary-care physician and hematologist.

\section{Features related to specific organ thrombosis}

As the clinical manifestations and severity vary widely according to the site of thrombosis, the WG decided to recommend organ-specific criteria. The subsequent paragraphs will discuss the current literature on long-term outcomes of various organ-specific thromboses, and the reasoning behind the suggested classification:

\section{Outcomes after neonatal and pediatric RVT}

Although isolated cases have been described in older children, RVT is most commonly seen in the neonatal age group. In a large literature review, although the definition was heterogeneous, irreversible damage was found in $70.6 \%$ of the affected kidneys, regardless of whether anticoagulant treatment was received [4]. At a median followup of 3.7 years, $20 \%$ of the patients developed hypertension, and $3 \%$ of the patients required renal replacement therapy or renal transplantation. There is a suggestion from previous studies that renal size and the laterality (unilateral vs. bilateral) may influence outcome [5]. On the basis of these data, a risk assignment score for RVT is suggested that is inexpensive, practical, objective, and easily available (Table 1). In combination with the risk assignment score and the estimated glomerular filtration rate (calculated with the Schwartz formula), outcomes following RVT and the need for renal replacement therapy (such dialysis or renal transplantation) should be noted. It is recommended that patients are assessed at 6-
Table 1 Outcomes after pediatric renal vein thrombosis

Recommended timeline: follow every 6 months after the event for 5 years

Diagnosis and severity to be assessed by using a combination of points (a) and (b) below

(a) Risk assignment score: a cumulative score should be assigned on the basis of the criteria below, and then be classified as Subclass A $($ score $\leq 4)$ and Subclass B (score $>4)$

\begin{tabular}{llll}
\hline Score & 0 & 1 & 2 \\
$\begin{array}{l}\text { Laterality } \\
\text { Renal atrophy }\end{array}$ & None & Unilateral & Bilateral \\
$\begin{array}{l}\text { Proteinuria (urine } \\
\text { protein/creatinine ratio) }\end{array}$ & $<0.2$ & $0.2-0.5$ & Bilateral \\
\begin{tabular}{l} 
Hypertension \\
\hline
\end{tabular} & None & Stage I & Stage II \\
\hline
\end{tabular}

(b) CKD staging (I-V) based on the glomerular filtration rate calculated with the Schwartz formula (see Supporting information for the reference) and the calculated risk of deterioration based on the risk assignment score. (Note: chronic kidney disease stage V denotes the need for renal replacement therapies such as dialysis and renal transplantation. In such cases, there is no longer a need to assign the risk assignment score)

month intervals after the episode of thrombosis for at least 5 years.

\section{Outcomes after neonatal or pediatric PVT}

PVT is a major cause of portal hypertension (PHT) in children, accounting for 5-20\% of all cases of PHT [6] (Table 2). In one neonatal study, PHT developed in 3$7 \%$ of cases on follow-up. PHT is usually defined clinically by the presence of a risk factor (e.g. PVT) and evidence of splenomegaly and/or portosystemic collaterals (e.g. esophageal varices) on abdominal imaging. Ultrasound with Doppler is the imaging modality of choice to provide supportive evidence for the presence of PHT, although the use of contrast-enhanced computed tomography (CT) and magnetic resonance venography has also been reported [7,8]. As there is no evidence for the efficacy of treatment for prevention of variceal bleeding

Table 2 Outcomes after pediatric portal vein thrombosis (PVT)

Recommended timeline: start screening at 6 months after the event (if the exact date is not known, then at the point of first detection), and then annually for 5 years

Diagnosis: portal hypertension is diagnosed in children with PVT by Doppler ultrasound showing splenomegaly and portosystemic collaterals

There is no validated system with which to assess the severity of portal hypertension caused by PVT. A suggested approach is as follows:

Mild or no portal hypertension: non-occlusive thrombus or occlusion in branch vein with no evidence of splenomegaly or clinically significant portosystemic shunting/collaterals Moderate: occlusion of main portal vein or multiple branch veins with evidence of splenomegaly and/or portosystemic shunting but without further complications

Severe: moderate plus evidence of complications such as significant thrombocytopenia, variceal bleeding, and encephalopathy 
in children, the role of screening endoscopy for variceal identification before a bleed occurs is controversial. After variceal bleeding, monthly endoscopy to enable treatment of varices (e.g. by endoscopic variceal ligation) is recommended until varices are obliterated, and then annually thereafter. Patients may present later with manifestations of PHT such as coincidental detection of splenomegaly, thrombocytopenia, or presentation with an acute variceal bleed. In one case series, PHT was identified at a mean of 5.7 years after the acute event [9]. In other reports, $80 \%$ of patients with PHT presented within the first 3 years of life [10]. As it is difficult to pinpoint the exact timing of the development of PVT, the WG recommends screening for PHT with ultrasound scan as soon as an occlusive main PVT or portal cavernoma is identified, and then annually thereafter. If physical examination and ultrasound screening in an otherwise stable patient do not suggest PHT after 5 years, it is reasonable to assume that PHT will not develop.

\section{Outcome after pediatric pulmonary arterial thrombosis (PE)}

Chronic thromboembolic pulmonary hypertension $(\mathrm{CTEPH})$ is a complication of acute symptomatic or asymptomatic PE, and is diagnosed when the mean pulmonary artery pressure is $\geq 25 \mathrm{mmHg}$, the pulmonary capillary wedge pressure is $\leq 15 \mathrm{mmHg}$ and the pulmonary vascular resistance index is $\geq 3 \mathrm{WU} \mathrm{m}^{-1}$ in the presence of chronic/organized thrombi/emboli after at least 6 months of anticoagulation [11]. It occurs in $0.57-9.1 \%$ of adults after acute PE, and the median time to diagnosis is 14.1 months. The exact incidence of pediatric CTEPH cannot be estimated, and it is probably underdiagnosed, owing to a lack of adequate follow-up data (Table S1).

Although the gold standard for diagnosis of CTEPH is pulmonary angiography, other non-invasive tests, such as echocardiography, the 6-min walk test, electrocardiogram, $\mathrm{N}$-terminal prohormone of brain natriuretic ppetide, and CT or magnetic resonance angiography, may be helpful in children who do not routinely undergo cardiac catheterization. A recent single-center study evaluated the long-term outcomes of PE in 58 children with PE who were followed for a median duration of 13.3 months. Echocardiography revealed that right ventricular dilatation was present in $36 \%$ and $13 \%$ of patients at followup of 6 months and 1 year, respectively.

The severity of CTEPH should be graded as per the World Health Organization classification [12], and followed in conjunction with a pediatric cardiologist/pulmonologist with experience in the management of CTEPH. As studies in adults suggest that most cases of CTEPH become apparent within 2 years of the PE $[13,14]$, the WG recommends that screening for CTEPH after PE may be performed 6 months after the diagnosis of PE, and annually thereafter for a period of 2 years (Table S1).

\section{Outcomes after thrombosis at other rare sites}

Pediatric BCS or hepatic venous outflow obstruction is rare. Possible outcomes after BCS include death, liver transplantation, persistent chronic liver disease, resolution of thrombus with anticoagulation or angioplasty, and persistence of thrombus with resolution of liver synthetic dysfunction and portal hypertensive complications. Although various indices have been used for prognostication in adults, the accuracy of these indices is poor (reference included in the Supporting information). None of the indices have been studied or validated in children with BCS, and we therefore recommend that patients with BCS should be followed up by a subspecialist with specific expertise in liver diseases. The WG also felt that recommendations about outcomes after thrombosis at other rare sites, such as mesenteric, pelvic or retinal veins, could not be made, owing to lack of information.

\section{Limitations of recommendations}

The purpose of this WG was to develop a systematic approach to measure outcomes after non-extremity VTE that are helpful in clinical care and for clinical trials studies. The overall quality of evidence was ' $2 \mathrm{C}$ ', as the methodological quality of supportive evidence was low, and there is uncertainty in the estimates of benefits vs. economic burden of screening tests [15]. The clinical benefit of these screening tools needs further validation.

\section{Addendum}

M. Rajpurkar reviewed the literature on outcomes after RVT, PE and thrombosis at other sites, wrote the manuscript, and coordinated the work with all of the authors. A. Sharathkumar reviewed the literature on outcomes after PE, and was involved in manuscript writing. S. Williams reviewed the literature on outcomes after PVT and PE, and reviewed and modified the manuscript. $\mathrm{K}$. Lau reviewed the literature on outcomes after RVT, and made recommendations on the follow-up of outcomes after RVT. S. C. Ling reviewed the literature on outcomes after PVT, and made recommendations on the follow-up of outcomes after PVT. A. Chan the SSC Chairman, who initiated the project, supervised all aspects of the manuscript writing, and made the final decision(s) on the recommendations. All authors reviewed and revised the manuscript. Additionally, the authors would like to thank P. Monagle for his guidance during the entire project. 


\section{Disclosure of Conflict of Interests}

The authors state that they have no conflict of interest.

\section{Supporting Information}

Additional Supporting Information may be found in the online version of this article:

Data S1. Outcomes after pulmonary artery thrombosis. Table S1. Outcomes after pulmonary arterial thrombosis (pulmonary embolism).

\section{References}

1 Goldenberg NA, Brandao L, Journeycake J, Kahn S, Monagle P, Revel-vilk S, Sharathkumar A, Chan AK. Definition of postthrombotic syndrome following lower extremity deep venous thrombosis and standardization of outcome measurement in pediatric clinical investigations. J Thromb Haemost 2012; 10: 477-80.

2 Revel-Vilk S, Brandao LR, Journeycake J, Goldenberg A, Monagle P, Sharathkumar A, Chan AK. Standardization of post-thrombotic syndrome definition and outcome assessment following upper venous system thrombosis in pediatric practice. J Thromb Haemost 2012; 10: 2182-5.

3 Kuhle S, Koloshuk B, Marzinotto V, Bauman M, Massicotte P, Andrew M, Chan A, Abdolell M, Mitchell L, Kuhle S, Koloshuk B, Marzinotto V, Bauman M, Massicotte P, Andrew M, Abdolell M, Mitchell L. A cross-sectional study evaluating post-thrombotic syndrome in children. Thromb Res 2003; 111: 227-33.

4 Lau KK, Stoffman JM, Williams S, McCusker P, Brandao L, Patel S, Chan AK. Neonatal renal vein thrombosis: review of the English-language literature between 1992 and 2006. Pediatrics 2007; 120: e1278-84.

5 Winyard PJ, Bharucha T, De Bruyn R, Dillon MJ, van't Hoff W, Trompeter RS, Liesner R, Wade A, Rees L. Perinatal renal venous thrombosis: presenting renal length predicts outcome. Arch Dis Child Fetal Neonatal Ed 2006; 91: F273-8.

6 Primignani M. Portal vein thrombosis, revisited. Dig Liver Dis 2010; 42: 163-70.

7 Pariente D, Franchi-Abella S. Paediatric chronic liver diseases: how to investigate and follow up? Role of imaging in the diagnosis of fibrosis. Pediatr Radiol 2010; 40: 906-19.

8 Webb LJ, Sherlock S. The aetiology, presentation and natural history of extra-hepatic portal venous obstruction. $Q \mathrm{~J} \mathrm{Med}$ 1979; 48: 627-39.

9 Alvarez F, Bernard O, Brunelle F, Hadchouel P, Odievre M, Alagille D. Portal obstruction in children. I. Clinical investigation and hemorrhage risk. J Pediatr 1983; 103: 696-702.

10 Sarin SK, Agarwal SR. Extrahepatic portal vein obstruction. Semin Liver Dis 2002; 22: 43-58.

11 Wilkens H, Lang I, Behr J, Berghaus T, Grohe C, Guth S, Hoeper MM, Kramm T, Kruger U, Langer F, Rosenkranz S, Schafers HJ, Schmidt M, Seyfarth HJ, Wahlers T, Worth H, Mayer E. Chronic thromboembolic pulmonary hypertension (CTEPH): updated Recommendations of the Cologne Consensus Conference 2011. Int J Cardiol 2011; 154(Suppl. 1): S54-60.

12 Simonneau G, Galie N, Rubin LJ, Langleben D, Seeger W, Domenighetti G, Gibbs S, Lebrec D, Speich R, Beghetti M, Rich S, Fishman A. Clinical classification of pulmonary hypertension. $J$ Am Coll Cardiol 2004; 43: 5S-12S.

13 Pengo V, Lensing AW, Prins MH, Marchiori A, Davidson BL, Tiozzo F, Albanese P, Biasiolo A, Pegoraro C, Iliceto S, Prandoni P. Incidence of chronic thromboembolic pulmonary hypertension after pulmonary embolism. $N$ Engl J Med 2004; 350: 2257-64.

14 Klok FA, van Kralingen KW, van Dijk AP, Heyning FH, Vliegen HW, Huisman MV. Prospective cardiopulmonary screening program to detect chronic thromboembolic pulmonary hypertension in patients after acute pulmonary embolism. Haematologica 2010; 95: 970-5.

15 Guyatt G, Gutterman D, Baumann MH, Addrizzo-Harris D, Hylek EM, Phillips B, Raskob G, Lewis SZ, Schunemann H. Grading strength of recommendations and quality of evidence in clinical guidelines: report from an American College of Chest Physicians task force. Chest 2006; 129: 174-81. 